\title{
On the formulation of balance laws for electromagnetic continua
}

\author{
David J. Steigmann \\ Department of Mechanical Engineering \\ 6133 Etcheverry Hall \\ University of California \\ Berkeley, CA. 94720
}

\begin{abstract}
Kovetz' formulation [1] of the system consisting of the linear momentum balance, momentof-momentum balance, energy balance and Clausius-Duhem inequality for electromagnetic media is derived from basic ideas in continuum mechanics and electromagnetism and shown thereby to be compatible with earlier formulations.
\end{abstract}

\section{Introduction}

Current work in the nonlinear electrodynamics of continua (e.g. [2]-[5]) reflects the influence of Kovetz' recent text [1], and for good reason. Kovetz presents the subject in a logical and deductive manner that appeals strongly to those trained in modern continuum mechanics. While contact with earlier work is largely absent, some may find the latter to be somewhat disjointed, and so will appreciate Kovetz' text for the organization it brings to the field. Even so, Kovetz' basic postulates concerning the laws of motion, conservation of energy and dissipation are difficult to motivate, and this appears to have led to some misinterpretations of his treatment vis a vis earlier work. In particular, his formulation of the balance law for energy and the Clausius-Duhem inequality are seemingly non-standard, and their consistency with alternative ideas recently revisited by Ericksen has been called into question [6, 7].

In the course of exploring the potential disparities, it emerges that Kovetz' treatment and that of Ericksen and others are in fact equivalent in respect of the global statements, and thus the local statements too if the fields are sufficiently smooth. Beyond this, to derive jump conditions at singular surfaces, including material boundaries, it is necessary to express the basic laws in conservation form to ensure the appropriate degree of regularity. Kovetz' formulation incorporates this requirement at the outset, but, as remarked by Ericksen [7], some of the alternative formulations do not. We conclude that Kovetz' formulation furnishes the mathematically appropriate alternative. The apparent discrepancies between his development and those of others [8-10] are due to the use of differing primitive concepts in constructing the foundations of the theory. We elaborate on these points by adopting a framework more familiar to workers trained in conventional continuum mechanics and using it to derive Kovetz' formulation. Along the way, some of the hypotheses on which the former is based are examined and their conceptual bases clarified. All of the concepts discussed are well-known in principle. They are collected here in anticipation of the need for a concise and pedagogically useful treatment. Notation currently standard in nonlinear continuum mechanics is used throughout [11]. 


\section{Charge conservation and Maxwell's equations}

A central tenet of electromagnetism is the principle of charge conservation, according to which charge can be added to or subtracted from a material region $P$ only through the mechanism of electrical conduction. Thus, if $Q(P, t)$ is the net charge, presumed to be an absolutely continuous function of volume, then [1]

$$
\frac{d}{d t} Q(P, t)=I(\partial P, t)
$$

where $I$, the current flowing through $\partial P$, is presumed to be an absolutely continuous function of surface area. This is then equivalent to

$$
\int_{P} q_{t} d v+\int_{\partial P} q \mathbf{v} \cdot \mathbf{n} d a=\int_{\partial P} \alpha d a
$$

where $q(\mathbf{x}, t)$ is the charge per unit volume, the subscript $t$ denoting a time derivative at fixed position $\mathbf{x} \in P, \alpha$ is the current flux per unit area, $\mathbf{v}$ is the material velocity, $\mathbf{n}$ is the exterior unit normal to $\partial P$ and use has been made of Reynolds' transport theorem. Adapting a theorem of Noll [12; Thm. 4], we conclude that if the volume distribution in (2) is bounded and $\partial P$ is smooth, then $\alpha$ is determined by the local tangent plane to $\partial P$ or, equivalently, by its local exterior unit normal $\mathbf{n}$. Cauchy's theorem [11] then yields this dependence in the form

$$
\alpha=-\mathcal{J} \cdot \mathbf{n},
$$

where $\mathcal{J}(\mathbf{x}, t)$, the conduction current, is independent of $\mathbf{n}$. The global statement obtained by combining (2) with (3) may be used with a standard procedure (e.g. $[13,14])$ to derive a jump condition at a singular surface, across which $q, \mathbf{v}$ or $\mathcal{J}$ may be discontinuous. For fields that are smooth and hence continuous, localization yields the classical form

$$
q_{t}+\operatorname{div} \mathbf{j}=0
$$

of the charge-conservation law, where

$$
\mathbf{j}=q \mathbf{v}+\mathcal{J}
$$

is the net current, the contribution $q \mathbf{v}$ being due to convection of charge by the material.

Two of Maxwell's equations arise from the integral of (4) in terms of a pair of vector potentials. Thus,

$$
q=\operatorname{div} \mathbf{d} \quad \text { and } \quad \mathbf{j}=\operatorname{curl} \mathbf{h}-\mathbf{d}_{t},
$$

where $\mathbf{d}(\mathbf{x}, t)$ and $\mathbf{h}(\mathbf{x}, t)$ are the electric displacement and magnetic field, respectively. It is well known that these equations do not determine $\mathbf{d}$ and $\mathbf{h}$ uniquely. However, this is of no consequence if these equations are used, as is common practice, to compute $q$ and $\mathbf{j}$ [1]. The second pair of Maxwell's equations are given, in local form, by

$$
\operatorname{div} \mathbf{b}=0 \quad \text { and } \quad \text { curle }-\mathbf{b}_{t}=\mathbf{0},
$$

where $\mathbf{e}(\mathbf{x}, t)$ is the electric field and $\mathbf{b}(\mathbf{x}, t)$ is the magnetic induction. These local forms follow from their global counterparts wherever the fields are smooth. However, the global equations also furnish 
jump conditions on singular surfaces. These are $[1,15,16]$

$$
\mathbf{n} \cdot[\mathbf{d}]=\sigma, \quad \mathbf{n} \times[\mathbf{h}]+u\left[\mathbf{d}^{\prime}\right]=\mathbf{k}, \quad \mathbf{n} \cdot[\mathbf{b}]=0, \quad \mathbf{n} \times[\mathbf{e}]-u[\mathbf{b}]=\mathbf{0}
$$

on a singular surface $S$ with local orientation $\mathbf{n}$, where square brackets are used to denote jumps, $\sigma$ is the surface charge per unit area of $S,\left[\mathbf{d}^{\prime}\right]$ is the projection of $[\mathbf{d}]$ onto the local tangent plane to $S, \mathbf{k}$ is the (tangential) surface current per unit length on $S$ and $u$ is the speed of propagation of $S$ in the direction of $\mathbf{n}$. For material surfaces this speed is given by the (continuous) restriction of $\mathbf{v} \cdot \mathbf{n}$ to $S$. It follows from (6) and (8) that $q$ and $\mathbf{j}$ are undefined on singular surfaces $[7,16]$. This has important implications for the balance laws considered in Sections 3 and 4.

Kovetz' text [1] contains an extensive account of the transformation properties of these fields under changes of the space-time frame of reference and their origins in postulates of invariance for the fourdimensional global forms of the equations.

When working with material media it is often convenient to replace Maxwell's equations $(6)_{2}$ and $(7)_{2}$ respectively by the equivalent relations $[1,15]$

$$
\mathcal{J}=\operatorname{curl} \mathcal{H}-\mathbf{d}^{*} \text { and } \operatorname{curlE}-\mathbf{b}^{*}=\mathbf{0}
$$

where

$$
\mathcal{H}=\mathbf{h}-\mathbf{v} \times \mathbf{d}, \quad \mathcal{E}=\mathbf{e}+\mathbf{v} \times \mathbf{b}
$$

and the superscript ${ }^{*}$ refers to the flux derivative

$$
\mathbf{a}^{*}=\mathbf{a}_{t}+\operatorname{div}(\mathbf{a} \otimes \mathbf{v})-(\nabla \mathbf{v}) \mathbf{a}=\mathbf{a}_{t}+(\operatorname{div} \mathbf{a}) \mathbf{v}-\operatorname{curl}(\mathbf{v} \times \mathbf{a})
$$

this having the convenient feature that [13]

$$
\frac{d}{d t} \int_{\omega} \mathbf{a} \cdot \mathbf{n} d a=\int_{\omega} \mathbf{a}^{*} \cdot \mathbf{n} d a
$$

for any material surface $\omega$.

It is conventional to split the charge and current into free and bound parts in which the former are regarded as supply terms and the latter as fields generated by the material in response to an electromagnetic field. Denoting these by subscripts ${ }_{f}$ and ${ }_{b}$, respectively, we have

$$
q=q_{f}+q_{b} \quad \text { and } \quad \mathbf{j}=\mathbf{j}_{f}+\mathbf{j}_{b}
$$

These generate the free and bound conduction currents $\mathcal{J}_{f, b}$, where

$$
\mathcal{J}_{f, b}=\mathbf{j}_{f, b}-q_{f, b} \mathbf{v}
$$

Commonly, the bound charge and current are interpreted in terms of a dipole model of matter [17], according to which there exist polarization and magnetization fields $\mathbf{p}(\mathbf{x}, t)$ and $\mathbf{m}(\mathbf{x}, t)$, vanishing outside matter, such that

$$
q_{b}=-\operatorname{div} \mathbf{p} \quad \text { and } \quad \mathbf{j}_{b}=\operatorname{curl} \mathbf{m}+\mathbf{p}_{t}
$$

These may be used to derive

$$
\mathcal{J}_{b}=\operatorname{curl} \mathcal{M}+\mathbf{p}^{*},
$$


where

$$
\mathcal{M}=\mathbf{m}+\mathbf{v} \times \mathbf{p} .
$$

Kovetz [1] notes that the dipole model is open to question and is in any case superfluous if the bound charge and current are required to satisfy the charge conservation law.

The linearity of Maxwell's equations implies that

$$
q_{f}=\operatorname{div} \mathbf{d}_{f}, \quad \mathbf{j}_{f}=\operatorname{curl} \mathbf{h}_{f}-\left(\mathbf{d}_{f}\right)_{t} \quad \text { and } \quad \mathcal{J}_{f}=\operatorname{curl} \mathcal{H}_{f}-\mathbf{d}_{f}^{*}
$$

where

$$
\mathbf{d}_{f}=\mathbf{d}+\mathbf{p}, \quad \mathbf{h}_{f}=\mathbf{h}-\mathbf{m} \quad \text { and } \quad \mathcal{H}_{f}=\mathcal{H}-\mathcal{M} .
$$

Jump conditions for these free fields and their bound counterparts, the polarization and magnetization, are similar to those already discussed for the total fields [1].

According to Lorentz' postulate [1] the total fields are connected by the aether relations

$$
\mathbf{d}=\varepsilon_{0} \mathbf{e} \quad \text { and } \quad \mathbf{h}=\mu_{0}^{-1} \mathbf{b},
$$

where $\varepsilon_{0}$ and $\mu_{0}$ are positive absolute constants such that $\varepsilon_{0} \mu_{0}=c^{-2}$, and where $c$ is the speed of light in vacuum. These are presumed valid in all space and in matter. It is well known that these relations are invariant under the Lorentz group of transformations rather than the Galilean transformations that preserve the equations of conventional non-relativistic Mechanics. However, these transformations are asymptotically coincident if the material velocity relative to a Galilean frame is much smaller than $c$ and if the diameter of the material body is bounded (see [1], eq. (12.9)).

\section{Balances of momenta}

The force acting on a charged particle in an electric field, relative to an inertial frame of reference $\Sigma$, is given by the product of the electric field vector and the charge. This is often taken as the definition of the electric field in terms of the force on a test charge. If the particle is moving at some velocity relative to $\Sigma$, then the force acting on it is given by the product of its charge with the field existing in its rest frame, relative to which it is momentarily at rest. At any fixed time the two frames are related by a Galilean transformation, the latter necessarily being different at different times if the particle velocity varies with time. The force on the particle relative to $\Sigma$ is called the Lorentz force.

For continua, we replace the charge by the charge density and use the Galilean transformation rule for charges, forces and electric fields to obtain the force on an arbitrary part $P$ of the region currently occupied by the material in the frame $\Sigma$ [1]. Thus, if $\mathbf{e}$ and $\mathbf{b}$ are the components of the electromagnetic field in $\Sigma$, then the Lorentz force exerted on the material occupying $P$ is

$$
\mathbf{f}(P, t)=\int_{P} q \mathcal{E} d v, \quad \text { where } \quad \mathcal{E}=\mathbf{e}+\mathbf{v} \times \mathbf{b},
$$

and $\mathbf{v}$ is the material velocity.

For particles there is no notion of conduction; thus, to preserve the analogy, we temporarily suppress the conduction current, obtaining

$$
\mathbf{f}(P, t)=\int_{P}(q \mathbf{e}+\mathbf{j} \times \mathbf{b}) d v .
$$


From this it is straightforward to use Maxwell's equations and the aether relations to derive a balance law. Assuming smooth fields and using (6), (20) and (22) together with Reynolds' transport theorem for the material region $P$, after some effort we derive $[6,8]$

$$
\mathbf{f}(P, t)=\int_{\partial P} \hat{\mathbf{T}} \mathbf{n} d a-\varepsilon_{0} \frac{d}{d t} \int_{P} \mathbf{e} \times \mathbf{b} d v
$$

where

$$
\hat{\mathbf{T}}=\mathbf{T}_{M}+\varepsilon_{0} \mathbf{e} \times \mathbf{b} \otimes \mathbf{v}
$$

and

$$
\mathbf{T}_{M}=\varepsilon_{0}\left(\mathbf{e} \otimes \mathbf{e}-\frac{1}{2} e^{2} \mathbf{I}\right)+\mu_{0}^{-1}\left(\mathbf{b} \otimes \mathbf{b}-\frac{1}{2} b^{2} \mathbf{I}\right), \quad \text { with } \quad e=|\mathbf{e}| \quad \text { and } \quad b=|\mathbf{b}|,
$$

is the Maxwell stress. We note that in the development leading from (22) to (23) no use was made of the assumption that the conduction current vanishes. In the general case [1]

$$
q \mathbf{e}+\mathbf{j} \times \mathbf{b}=q \mathcal{E}+\mathcal{J} \times \mathbf{b},
$$

so that eqs. (22) and (23) remain valid under this replacement. The term $\mathcal{J} \times \mathbf{b}$ may thus be interpreted as a force density due to conduction. We adopt (23) as the global electromagnetic momentum balance for continua, with the Lorentz force acting on the material given by

$$
\mathbf{f}(P, t)=\int_{P}(q \mathcal{E}+\mathcal{J} \times \mathbf{b}) d v
$$

Adopting a convention common in continuum mechanics we regard (23) as being valid, with $\mathbf{f}(P, t)$ interpreted as the Lorentz force, whether or not the fields are smooth. In particular, for fields that suffer jump discontinuities, the limit of the right-hand side of (27) obtained by collapsing $P$ onto a singular surface $S$ (in the manner described, for example, in [13], [14]) need not vanish. Our convention thus addresses the objection (see $[7,16]$ ) that volume distributions of charge and current, being related to the electromagnetic field via (6) and (20), are not defined on singular surfaces. Indeed, it is difficult to conceive of any alternative convention. For example, one might suppose that discontinuities in the fields make a contribution of the form (27) to the Lorentz force, with $P$ replaced by $S$ and with $q$ and $\mathcal{J}$ replaced respectively by an associated surface charge and conduction current, and that such a contribution could simply be added to (27), with $P$ replaced by $P \backslash S$, the result serving as the left-hand side of (23). However, since jumps in $\mathcal{E}$ and $\mathbf{b}$ occur across $S$ the specification of such a force entails a degree of ambiguity. The present interpretation for fields with singular surfaces is essentially Ericksen's [7] (see also [16]).

The conventional conservation law for linear momentum requires that the rate of change of the linear momentum of any part of the body be balanced by the net force. Thus,

$$
\frac{d}{d t} \int_{P} \rho \mathbf{v} d v=\int_{\partial P} \overline{\mathbf{t}} d a+\int_{P} \rho \overline{\mathbf{b}} d v+\mathbf{f}(P, t),
$$

where $\rho$ is the mass density, $\overline{\mathbf{t}}$ is a surface force density and $\overline{\mathbf{b}}$ is a volume force density, the latter being assigned in the frame $\Sigma$. If the volume distributions in (28) are bounded then the Noll and Cauchy theorems yield

$$
\overline{\mathbf{t}}=\overline{\mathbf{T}} \mathbf{n}
$$


where $\overline{\mathbf{T}}(\mathbf{x}, t)$ is independent of $\mathbf{n}$. To ensure that the conditions of Noll's theorem are met, we substitute (23) into (28) and re-write the latter as

$$
\frac{d}{d t} \int_{P} \rho \mathbf{g} d v=\int_{\partial P} \mathbf{t} d a+\int_{P} \rho \overline{\mathbf{b}} d v
$$

where

$$
\mathbf{g}=\mathbf{v}+\left(\varepsilon_{0} \mathbf{e} \times \mathbf{b} / \rho\right) \quad \text { and } \quad \mathbf{t}=\overline{\mathbf{t}}+\hat{\mathbf{T}} \mathbf{n} .
$$

Replacing $\mathbf{v}$ by $\mathbf{g}$ in the statement of Noll's theorem [12], we are led to conclude that $\mathbf{t}$ depends on $\mathbf{n}$; by Cauchy's theorem to conclude that

$$
\mathbf{t}=\mathbf{T n}
$$

where $\mathbf{T}(\mathbf{x}, t)$ is independent of $\mathbf{n}$, and thus finally to (29), with

$$
\overline{\mathbf{T}}=\mathbf{T}-\hat{\mathbf{T}} .
$$

Kovetz [1] proposes (30) as the primitive statement of the balance of linear momentum, leaving $\mathbf{g}$ and $\mathbf{T}$ to be determined by constitutive assumptions together with additional laws of balance and dissipation to be discussed below. For the examples that he considers, pertaining to viscous and inviscid fluids and elastic solids, $\mathbf{g}$ is always found to reduce to $(31)_{1}$. Here, we assume that $(31)_{1}$ holds in general, relying for justification on the passage from (28) to (30). The jump condition associated with (30) is given by eq. (51.19) of [1]. It is not equivalent to that obtained by using (27) with (28), which is inappropriate due to the potential unboundedness of the integrand in (27) on singular surfaces. For material boundaries, Kovetz' jump condition reduces to $[\mathbf{T}] \mathbf{n}=\mathbf{0}$, where $\mathbf{n}$ is the unit normal to the surface directed from the interior to the exterior. Thus,

$$
\mathbf{t}_{a}+\mathbf{T}_{M}^{+} \mathbf{n}=\mathbf{T}^{-} \mathbf{n}
$$

where the superscripts ${ }^{ \pm}$identify exterior and interior limits, respectively, and $\mathbf{t}_{a}$ is the applied traction, this being equal to the exterior limit $\overline{\mathbf{t}}^{+}=\overline{\mathbf{T}}^{+} \mathbf{n}$.

The equations of motion follow by localizing (28) or (30) in regions of smoothness. Using (29) in the first alternative yields

$$
\rho \dot{\mathbf{v}}=\operatorname{div} \overline{\mathbf{T}}+\rho \overline{\mathbf{b}}+q \mathcal{E}+\mathcal{J} \times \mathbf{b}
$$

given by Ericksen [6, 7], while the second alternative yields Kovetz' equation

$$
\rho \dot{\mathbf{g}}=\operatorname{div} \mathbf{T}+\rho \overline{\mathbf{b}}
$$

These equations are equivalent by construction. Conservation of mass is used here and elsewhere, usually without explicit mention. Also, superposed dots refer to the material derivative, the time derivative following a fixed material point.

With (35) satisfied in an arbitrary region $P$ of sufficient smoothness in the relevant fields, the moment-of-momentum balance

$$
\frac{d}{d t} \int_{P}\left(\mathbf{x}-\mathbf{x}_{0}\right) \times \rho \mathbf{v} d v=\int_{\partial P}\left(\mathbf{x}-\mathbf{x}_{0}\right) \times \overline{\mathbf{T}} \mathbf{n} d a+\int_{P}\left(\mathbf{x}-\mathbf{x}_{0}\right) \times(\rho \overline{\mathbf{b}}+q \mathcal{E}+\mathcal{J} \times \mathbf{b}) d v,
$$


with $\mathbf{x}_{0}$ an arbitrary fixed position, leads, following the standard procedure, to

$$
\overline{\mathbf{T}}=\overline{\mathbf{T}}^{t}
$$

Substitution of (33) with (24) furnishes a restriction equivalent to eq. (51.21) of [1], which in turn follows from (36) and

$$
\frac{d}{d t} \int_{P}\left(\mathbf{x}-\mathbf{x}_{0}\right) \times \rho \mathbf{g} d v=\int_{\partial P}\left(\mathbf{x}-\mathbf{x}_{0}\right) \times \mathbf{T} \mathbf{n} d a+\int_{P}\left(\mathbf{x}-\mathbf{x}_{0}\right) \times \rho \overline{\mathbf{b}} d v,
$$

proposed by Kovetz [1] as the primitive form of the moment-of-momentum balance law.

\section{Balance of energy}

The Lorentz force satisfies a power identity following from Maxwell's equations. This is the content of Poynting's theorem. For smooth fields, the power of the Lorentz force is

$$
\Pi_{L}=\int_{P}(q \mathcal{E}+\mathcal{J} \times \mathbf{b}) \cdot \mathbf{v} d v=\int_{P}(\mathbf{j} \cdot \mathbf{e}-\mathcal{J} \cdot \mathcal{E}) d v,
$$

the second equality deriving from the combination of (5), (10) 2 and (26). Poynting's theorem follows by using Maxwell's equations, the aether relations, the identity

$$
\operatorname{div}(\mathbf{h} \times \mathbf{e})=\mathbf{h} \cdot \operatorname{curl} \mathbf{e}-\mathbf{e} \cdot \operatorname{curl} \mathbf{h},
$$

and Reynolds' transport theorem to eliminate the integral of $\mathbf{j} \cdot \mathbf{e}$. Thus [7],

$$
\Pi_{L}+\int_{P} \mathcal{J} \cdot \mathcal{E} d v=\int_{P} \mathbf{j} \cdot \mathbf{e} d v=\int_{\partial P}\left[\frac{1}{2}\left(\varepsilon_{0} e^{2}+\mu_{0}^{-1} b^{2}\right) \mathbf{v}-\mathbf{e} \times \mathbf{h}\right] \cdot \mathbf{n} d a-\frac{d}{d t} \int_{P} \frac{1}{2}\left(\varepsilon_{0} e^{2}+\mu_{0}^{-1} b^{2}\right) d v
$$

For reasons discussed in connection with (23), we take the outer equality to be the global statement of Poynting's theorem whether or not singular surfaces are present. This facilitates the derivation of the relevant jump condition, avoiding the potential unboundedness of $\mathbf{j}$ on a singular surface.

For our present purposes it is convenient to recast this in a form involving $\mathcal{E} \times \mathcal{H}$. With some algebra based on (10), (20) and (25), the appropriate connection is found to be

$$
-\mathbf{e} \times \mathbf{h} \cdot \mathbf{n}+\frac{1}{2}\left(\varepsilon_{0} e^{2}+\mu_{0}^{-1} b^{2}\right) \mathbf{v} \cdot \mathbf{n}=-\mathcal{E} \times \mathcal{H} \cdot \mathbf{n}+\mathbf{T}_{M} \mathbf{v} \cdot \mathbf{n}+\varepsilon_{0}[(\mathbf{v} \otimes \mathbf{e} \times \mathbf{b}) \mathbf{v}] \cdot \mathbf{n},
$$

yielding

$$
\Pi_{L}=-\int_{P} \mathcal{J} \cdot \mathcal{E} d v+\int_{\partial P}(\hat{\mathbf{T}} \mathbf{n} \cdot \mathbf{v}-\mathcal{E} \times \mathcal{H} \cdot \mathbf{n}) d a-\frac{d}{d t} \int_{P} \frac{1}{2}\left(\varepsilon_{0} e^{2}+\mu_{0}^{-1} b^{2}\right) d v .
$$

The global balance of energy is taken to be [8]

$$
\frac{d}{d t} \bar{E}=\Pi+H
$$

where

$$
\bar{E}=\int_{P} \rho \bar{\epsilon} d v
$$

is the energy of the material occupying the region $P, \bar{\epsilon}$ is the energy per unit mass,

$$
\Pi=\bar{\Pi}+\Pi_{L}
$$


with

$$
\bar{\Pi}=\int_{\partial P} \overline{\mathbf{T}} \mathbf{n} \cdot \mathbf{v} d a+\int_{P} \rho \overline{\mathbf{b}} \cdot \mathbf{v} d a,
$$

is the power of the forces acting on $P$, and

$$
H=\bar{H}+H_{J},
$$

with

$$
\bar{H}=\int_{P} \rho \bar{r} d v+\int_{\partial P} \bar{h} d a
$$

and

$$
H_{J}=\int_{P} \mathcal{J} \cdot \mathcal{E} d v
$$

is the total heating imparted to $P$. Here $H_{J}$ is the Joule heating, while $\bar{r}$ and $\bar{h}$ are the conventional volumetric heating and heat flux, respectively. The net supply to the body from the electromagnetic field is thus given by [8]

$$
\Pi_{L}+H_{J}=\int_{P} \mathbf{j} \cdot \mathbf{e} d v .
$$

Further, the Noll and Cauchy theorems, applied to (45), yield the existence of a vector field $\overline{\mathbf{q}}(\mathbf{x}, t)$ such that

$$
\bar{h}=-\overline{\mathbf{q}} \cdot \mathbf{n} .
$$

Thus,

$$
\frac{d}{d t} \bar{E}=\int_{\partial P} \overline{\mathbf{T}} \mathbf{n} \cdot \mathbf{v} d a-\int_{\partial P} \overline{\mathbf{q}} \cdot \mathbf{n} d a+\int_{P} \rho(\overline{\mathbf{b}} \cdot \mathbf{v}+\bar{r}) d v+\int_{P} \mathbf{j} \cdot \mathbf{e} d v
$$

The contribution of the power of the Lorentz force to the total power is easily motivated. However, the origin of the Joule heating is less obvious. We have not found a convincing argument in support of (51) in the continuum mechanics literature, and thus present a heuristic argument inspired by that found in some physics texts (eg. [17]). To this end we regard the conduction current $\mathcal{J}$ as arising from the motion of mobile electrons moving relative to the material, at average relative velocity $\mathbf{u}$. These generate a charge density $p=N M e$, where $N$ is the number of molecules per unit volume, $M$ is the number of mobile electrons per molecule, and $e$ is the electronic charge. Thus, $\mathcal{J}=p \mathbf{u}$. From (5), the total current is

$$
\mathbf{j}=q \mathbf{v}+p \mathbf{u}=(q-p) \mathbf{v}+p \mathbf{w},
$$

where $\mathbf{w}=\mathbf{u}+\mathbf{v}$ is the absolute velocity of $p$ relative to the frame $\Sigma$. If $\{\mathbf{e}, \mathbf{b}\}$ is the electromagnetic field in $\Sigma$, then the Lorentz force on the charge $q-p$, moving at velocity $\mathbf{v}$, is $(q-p)(\mathbf{e}+\mathbf{v} \times \mathbf{b})$. The associated power is $(q-p)(\mathbf{e}+\mathbf{v} \times \mathbf{b}) \cdot \mathbf{v}=(q-p) \mathbf{e} \cdot \mathbf{v}$. Similarly, the Lorentz force on $p$ and the power generated by it are $p(\mathbf{e}+\mathbf{w} \times \mathbf{b})$ and $p \mathbf{e} \cdot \mathbf{w}$, respectively. The net power density is thus given by

$$
(q-p) \mathbf{e} \cdot \mathbf{v}+p \mathbf{e} \cdot \mathbf{w}=q \mathbf{v} \cdot \mathbf{e}+p \mathbf{u} \cdot \mathbf{e}=\mathbf{j} \cdot \mathbf{e}
$$

The interpretation of this as the sum of the power of a force acting against a material velocity and a heating supply (cf. (52)) is thus seen to be merely a matter of convention.

On combining (24) and (33), and substituting $(42)_{2}$ in place of the right-hand side of (52), the energy balance (54) assumes the form

$$
\frac{d}{d t} E=\int_{P} \rho \overline{\mathbf{b}} \cdot \mathbf{v} d v+\int_{\partial P} \mathbf{T n} \cdot \mathbf{v} d a+\int_{P} \rho \bar{r} d v-\int_{\partial P}\left(\mathbf{q}+\mathcal{E} \times \mathcal{H}_{f}\right) \cdot \mathbf{n} d a
$$


where

$$
E=\int_{P} \rho \epsilon d v
$$

with

$$
\epsilon=\bar{\epsilon}+\frac{1}{2}\left(\varepsilon_{0} e^{2}+\mu_{0}^{-1} b^{2}\right) / \rho
$$

and

$$
\mathbf{q}=\overline{\mathbf{q}}+\mathcal{E} \times \mathcal{M}
$$

This may be viewed as the balance of energy for the material and the electromagnetic field, taken together, occupying the region $P$. Eq. (57) is entirely equivalent to the balance law postulated by Kovetz [1; eq. (54.5)]. It yields a well-defined jump condition at singular surfaces, equivalent to eq. (54.6) of [1]. It is also identical, apart from the free-field term, to the balance law discussed by Ericksen $[6,7]$, who adopts a version of $(60)$ as the net heat flux vector. Motivation for the interpretation of $(60)$ as the net heat flux is furnished by an argument put forth by Liu and Müller [10; Sect. 6].

Localization of (57) in a region of smoothness furnishes

$$
\rho \dot{\epsilon}=\rho \overline{\mathbf{b}} \cdot \mathbf{v}+\operatorname{div}\left(\mathbf{T}^{t} \mathbf{v}\right)+\rho \bar{r}-\operatorname{div}\left(\mathbf{q}+\mathcal{E} \times \mathcal{H}_{f}\right) .
$$

Alternatively, and equivalently, localization of (54) yields

$$
\rho(\bar{\epsilon})^{\cdot}=\rho \overline{\mathbf{b}} \cdot \mathbf{v}+\operatorname{div}\left(\overline{\mathbf{T}}^{t} \mathbf{v}\right)+\rho \bar{r}+\mathbf{j} \cdot \mathbf{e}-\operatorname{div} \overline{\mathbf{q}} .
$$

However, the jump condition derived from (54) is not meaningful due the possible nonexistence of $\mathbf{j}$ on surfaces of discontinuity (cf. (6), (8) and [7]). Using the equation of motion (35) to eliminate the power of the body force, together with the local form of $(40)_{2}$, we derive the energy balance for smooth fields in the form given by Ericksen [6, 7]:

$$
\rho(\bar{u})^{\cdot}=\overline{\mathbf{T}} \cdot \nabla \mathbf{v}+\mathcal{J} \cdot \mathcal{E}+\rho \bar{r}-\operatorname{div} \overline{\mathbf{q}},
$$

where

$$
\bar{u}=\bar{\epsilon}-\frac{1}{2}|\mathbf{v}|^{2}
$$

is the internal energy of the material per unit mass. We split the conduction current into free and bound parts. Thus, $\mathcal{J}=\mathcal{J}_{f}+\mathcal{J}_{b}$. Maxwell's equations in the form $(9)_{2}$ and (16), when combined with an identity similar to (41), lead to

$$
\mathcal{J}_{b} \cdot \mathcal{E}=-\operatorname{div}(\mathcal{E} \times \mathcal{M})+\mathcal{E} \cdot \mathbf{p}^{*}-\mathcal{M} \cdot \mathbf{b}^{*}
$$

and thus permit us to replace (63) with

$$
\rho(\bar{u})^{\cdot}=\overline{\mathbf{T}} \cdot \nabla \mathbf{v}+\mathcal{J}_{f} \cdot \mathcal{E}+\rho \bar{r}+\mathcal{E} \cdot \mathbf{p}^{*}-\mathcal{M} \cdot \mathbf{b}^{*}-\operatorname{div} \mathbf{q},
$$

where $\mathbf{q}$ is given by $(60)$.

\section{Clausius-Duhem inequality}


Following Liu and Müller [10], Ericksen [6] proposes the Clausius-Duhem inequality in a form equivalent to

$$
\rho \theta \dot{\eta} \geq \rho \bar{r}-\theta \operatorname{div}(\mathbf{q} / \theta)
$$

for smooth fields, where $\eta$ is the entropy per unit mass, $\theta$ is the empirical temperature, and $\mathbf{q}$ is given by (60). Using (63) to eliminate $\rho \bar{r}-\operatorname{div} \mathbf{q}$, this may be recast as

$$
\rho\left[\theta \dot{\eta}-(\bar{u})^{\cdot}\right]+\overline{\mathbf{T}} \cdot \nabla \mathbf{v}+\mathcal{J}_{f} \cdot \mathcal{E}+\mathcal{E} \cdot \mathbf{p}^{*}-\mathcal{M} \cdot \mathbf{b}^{*}-\theta^{-1} \mathbf{q} \cdot \nabla \theta \geq 0
$$

Since the work of Coleman and Noll [18], the Clausius-Duhem inequality has been used as a device to derive restrictions on constitutive equations. These are typically assumed to depend on the local point values of various fields deemed to be relevant. Since smooth global fields can always be fitted to these point values, it suffices to confine attention to smooth fields in the consideration of such restrictions.

To effect this program here, it is convenient to write (68) in a form that involves material derivatives instead of flux derivatives. This is achieved by substituting

$$
\mathcal{E} \cdot \mathbf{p}^{*}=\mathcal{E} \cdot \dot{\mathbf{p}}+[(\mathcal{E} \cdot \mathbf{p}) \mathbf{I}-\mathcal{E} \otimes \mathbf{p}] \cdot \nabla \mathbf{v} \quad \text { and } \quad \mathcal{M} \cdot \mathbf{b}^{*}=\mathcal{M} \cdot \dot{\mathbf{b}}+[(\mathcal{M} \cdot \mathbf{b}) \mathbf{I}-\mathcal{M} \otimes \mathbf{b}] \cdot \nabla \mathbf{v}
$$

We define

$$
\varphi=\bar{u}-\theta \eta-\rho^{-1} \mathcal{E} \cdot \mathbf{p}
$$

and use conservation of mass in the form

$$
\dot{\rho}=-\rho \mathbf{I} \cdot \nabla \mathbf{v}
$$

to reduce $(68)$ to

$$
-\rho(\dot{\varphi}+\eta \dot{\theta})+\mathcal{J}_{f} \cdot \mathcal{E}-\mathbf{p} \cdot \mathcal{E} \cdot \mathcal{M} \cdot \dot{\mathbf{b}}+\boldsymbol{\tau} \cdot \nabla \mathbf{v}-\theta^{-1} \mathbf{q} \cdot \nabla \theta \geq 0,
$$

where

$$
\boldsymbol{\tau}=\overline{\mathbf{T}}-(\mathcal{M} \cdot \mathbf{b}) \mathbf{I}-\mathcal{E} \otimes \mathbf{p}+\mathcal{M} \otimes \mathbf{b}
$$

From (24) and (33) the latter may be written in the alternative form

$$
\boldsymbol{\tau}=\mathbf{T}+\frac{1}{2}\left(\varepsilon_{0} e^{2}+\mu_{0}^{-1} b^{2}-2 \mathcal{M} \cdot \mathbf{b}\right) \mathbf{I}-\varepsilon_{0} \mathbf{e} \otimes \mathbf{e}-\mu_{0}^{-1} \mathbf{b} \otimes \mathbf{b}-\mathcal{E} \otimes \mathbf{p}+\mathcal{M} \otimes \mathbf{b}-\varepsilon_{0} \mathbf{e} \times \mathbf{b} \otimes \mathbf{v}
$$

by which (72) is seen to be precisely Kovetz' statement of the Clausius-Duhem inequality [19; eqs. (14.11) and (14.12)]. This is used in $[1,19]$ to deduce the basic structure of constitutive equations for polarized and magnetized fluids and elastic solids (see also [20]).

Acknowledgment: This note was inspired by a course Electrodynamics of Continuous Media delivered by the writer at the University of California.

\section{References:}

1. A. Kovetz, 2000, Electromagnetic Theory. Oxford University Press. 
2. A. Dorfmann and R.W. Ogden, 2003, Magnetoelastic modelling of elastomers. Euro. J. Mech. A/Solids 22, 497-507.

3. S.V. Kankanala and N. Triantafyllidis, 2004, On finitely strained magnetorheological elastomers. J. Mech. Phys. Solids 52, 2869-2908.

4. D.J. Steigmann, 2004, Equilibrium theory for magnetic elastomers and magnetoelastic membranes. Int. J. Non-linear Mech. 39, 1193-1216.

5. M. Barham, D.J. Steigmann, M. McElfresh and R.E. Rudd, 2007, Finite deformation of a pressurized magnetoelastic membrane in a stationary dipole field. Acta Mechanica (in press).

6. J.L. Ericksen, 2007, Magnetizable and polarizable materials. Math. Mech. Solids (in press).

7. J.L. Ericksen, 2007, On formulating and assessing continuum theories of electromagnetic fields in elastic materials. J. Elasticity (in press).

8. C. Truesdell and R. Toupin, 1960, The classical field theories, in: S. Flügge (Ed.), Handbuch der Physik, Vol. III/1, Springer, Berlin.

9. R. Toupin, 1963, A dynamical theory of elastic dielectrics. Int. J. Engng. Sci. 1, 101-126.

10. I.-S. Liu and I. Müller, 1972, On the thermodynamics and thermostatics of fluids in electromagnetic fields. Archive for Rational Mechanics and Analysis 46, 149-176.

11. M.E. Gurtin, 1981, An Introduction to Continuum Mechanics. Academic Press, Orlando.

12. W. Noll, 1959, The foundations of classical mechanics in the light of recent advances in continuum mechanics, in: Foundations of the Axiomatic Method, with Special Reference to Geometry and Physics (Berkeley 1957), pp. 266-281. North-Holland, Amsterdam.

13. P. Chadwick, 1999, Continuum Mechanics: Concise Theory and Problems. Dover, New York.

14. I.-S. Liu, 2002, Continuum Mechanics. Springer, Berlin.

15. A.C. Eringen and G. Maugin, 1989, Electrodynamics of Continua, Vol. 1. Springer, New York.

16. K. Hutter and A.F. van de Ven, 1978, Field Matter Interactions in Thermoelastic Solids, Lecture Notes in Physics, Vol. 88. Springer, Berlin

17. D.J. Griffiths, 1989, Introduction to Electrodynamics, 2nd edn. Prentice Hall, Englewood Cliffs, NJ.

18. B.D. Coleman and W. Noll, 1963, The thermodynamics of elastic materials with heat conduction and viscosity. Archive for Rational Mechanics and Analysis 13, 167-178.

19. A. Kovetz, 1990, The Principles of Electromagnetic Theory. Cambridge University Press.

20. B.D. Coleman and E.H. Dill, 1971, Thermodynamic restrictions on the constitutive equations of electromagnetic materials. ZAMP 22, 691-702. 